\title{
Statistics in School Mathematics
}

\author{
Mária Jurečková ${ }^{1}$, Lucia Csachová \\ Catholic University in Ružomberok, Slovak Republic
}

\begin{abstract}
Nowadays, information in the form of tables, graphs and charts is common not only in news, politics and advertising, but also in many other areas of society. Given that many claims are used to misrepresent or obfuscate real data, there is a need to build people's ability to think about data credibility. The competence to correctly orientate and critically assess the presented data, socalled the statistical literacy, is therefore becoming increasingly important. Obtaining such a competence takes a long time, and it is therefore necessary to start building statistical literacy already in primary and secondary education. The article is focused on monitoring the level of statistical literacy of pupils of 9th grade at primary schools in the Slovak Republic. The results of several years testing of pupils were used for this purpose. In addition to quantitative data analysis, attention is also paid to qualitative analysis of the problems in the field of statistics that were most difficult for pupils. We aim to apply the knowledge from the conducted research to training of future teachers of mathematics, but also to courses within the lifelong professional education of elementary and secondary school teachers.
\end{abstract}

Keywords: education; statistical analysis; statistical literacy; testing 